\title{
Performance Evaluation of Cyclic Stability and Capacitance of Manganese Oxide Modified Graphene Oxide Nanocomposite for Potential Supercapacitor Applications
}

\author{
R. Ranjith Kumar, ${ }^{1}$ S. Thanigaivel, ${ }^{2}$ Nibedita Dey, ${ }^{2}$ A. K. Priya $\left(\mathbb{D},{ }^{3}\right.$ Alagar Karthick $\mathbb{D},{ }^{4,5}$ \\ V. Mohanavel $\mathbb{D},{ }^{6}$ S. Kannadhasan, ${ }^{7}$ M. Muhibbullah $\mathbb{D}^{8},{ }^{8}$ and Sameh M. Osman ${ }^{9}$ \\ ${ }^{1}$ Department of Civil Engineering, SRM Institute of Science and Technology, NCR Campus, Modinagar, -201204. Uttar Pradesh, \\ Ghaziabad, Delhi, India \\ ${ }^{2}$ Saveetha School of Engineering, Department of Biotechnology, Saveetha Institute of Medical and Technical Sciences, Thandalam, \\ 602105, Chennai, Tamil Nadu, India \\ ${ }^{3}$ Department of Civil Engineering, KPR Institute of Engineering and Technology, -641407, Coimbatore, Tamilnadu, India \\ ${ }^{4}$ Renewable Energy Lab, Department of Electrical and Electronics Engineering, KPR Institute of Engineering and Technology, \\ Coimbatore 641407, Tamil Nadu, India \\ ${ }^{5}$ Departamento de Quimica Organica, Universidad de Cordoba, EdificioMarie Curie (C-3), Ctra Nnal IV-A, Km 396, \\ E14014 Cordoba, Spain \\ ${ }^{6}$ Chennai-Centre for Materials Engineering and Regenerative Medicine, Bharath Institute of Higher Education and Research, \\ 600073 Tamilnadu, India \\ ${ }^{7}$ Department of Electronics and Communication Engineering, Cheran College of Engineering, -639111, Karur, Tamilnadu, India \\ ${ }^{8}$ Department of Electrical and Electronic Engineering, Bangladesh University, Dhaka 1207, Bangladesh \\ ${ }^{9}$ Chemistry Department, College of Science, King Saud University, P.O. Box 2455, Riyadh 11451, Saudi Arabia
}

Correspondence should be addressed to M. Muhibbullah; m.muhibbullah@bu.edu.bd

Received 5 October 2021; Revised 3 December 2021; Accepted 17 December 2021; Published 22 January 2022

Academic Editor: Awais Ahmed

Copyright (c) 2022 R. Ranjith Kumar et al. This is an open access article distributed under the Creative Commons Attribution License, which permits unrestricted use, distribution, and reproduction in any medium, provided the original work is properly cited.

Supercapacitors are a revolutionary type of energy storage device. They must be able to charge and discharge quickly while maintaining a high energy density. A storage material's cyclic stability is a desirable feature. The type of electrode materials employed for the specific study design affects supercapacitor performance. Manganese dioxide has long been regarded as one of the best and most abundant materials in nature, having a potentially high specific capacitance. They also offer a wider potential range, more electroactivity, and are more environmentally friendly. However, because of its decreased volume expansion and low conductivity, it is difficult to use as a capacitor material. As a result, carbon-based porous films and supports can be employed to produce critical composites to overcome the current shortcoming. These nanoparticle-based materials will have improved electrical conductivity and a large surface area. Graphene oxide (GO) has a high surface area, thermal stability, and porosity. As an electrode material, many types of $\mathrm{MnO}$ /carbon-based materials have been widely used in supercapacitors. Their overall performance is influenced by their construction processes, metal ratios, electrolyte medium, and voltage factors. Microwave technology was chosen as a cost-efficient and effective alternative to expensive and laborious techniques for fabricating $\mathrm{MnO} 2 / \mathrm{GO}$ composites. The production procedure of a supercapacitor has been explored in this study using MnO2-GO composite materials. Using the electrochemical deposition process, the nanocomposite materials of $\mathrm{MnO}$-GO are significantly deposited on the stainless steel (SS) substrate material. Galvanostatic charge-discharge techniques and cyclic voltammetry (CV) analytical methods were used to investigate the storage and cycle ability of supercapacitors. The composite $\mathrm{MnO} 2-\mathrm{GO}$ supercapacitor has a higher electrochemical capacitance based on these findings. 


\section{Introduction}

There is a great demand for both energy (conventional and nonconventional) resources due to the increase in living standards. For that reason, the electrochemical energy storages are obtained to be the substitute for this problem [1, 2]. A supercapacitor (SC), formerly electric double layer capacitor (EDLC), is the generic term for the electrochemical capacitor (ECs) family. A conventional solid dielectric is not present in SCs. Two storage principles determine the capacitance value of an EC, which both indivisibly contribute to the total capacitance $[1,3]$. In this day and age, consumption of energy in the form of fossil fuels like oil, coal, and natural gas has drastically increased. This adversely affects the world and the environment in ways like global warming, ecological destruction, and endangering life forms. Utilization of renewable energy is the need of the hour as it is ecofriendly. Countries have allotted funds and manpower exclusively to develop ways to use and create energy. Solar, tidal, hydrothermal, geothermal, and wind energy have played their share in alleviating major problems related to energy and environmental conservation all around the world [3, 4]. However, clean energy is very limited and cannot be directly applied to many applications. This is due to the natural conditions and the shortfall of resources; it greatly depends on the types of sources used and causes poor stability as well as tunability with regard to electricity generation. Hence, trustworthy electrochemical systems are encouraged to address efficient conversion, utilization, and storage of above sources [4]. Supercapacitors also known as electrochemical capacitors are the next-generation storage devices. They are popular for their green and clean nature of fabrication and implementation [5]. They also have a greater capacitance potential with higher power output and shelf life when compared to lithium-ion batteries [6]. Along with simple structure, they possess faster charging and pollution free effects while manufacturing. They can be easily applied to portable electronics, hybrid electric locomotives, data backup, etc. [7].

Due to their compact structures, high surface area, unique chemical, physical, and mechanical capabilities, nanomaterials have gotten a lot of interest in recent years. The ability to produce and process NMs is the first milestone in nanotechnology for exploring novel physical features and understanding possible uses of NMs. The NMs are also epic in structure and have features that can be adjusted. As a result of this development, NM has been useful in realworld applications $[2,8]$.

Manganese oxide $\left(\mathrm{MnO}_{2}\right)$ offers the benefit of relatively cost-effective, high theoretical capacitance $\left(\sim 1300 \mathrm{Fg}^{-1}\right)$, and environmentally compatible [9]. $\mathrm{MnO}_{2}$ is used for catalysts, sensors, lithium batteries, and alkaline $\mathrm{MnO}_{2} / \mathrm{Zn}$ cell applications. The advancements required in generating active materials mainly concern with overall stability, high reversible capacitance, structural flexibility, improved cation diffusion rate under high charge-discharge condition, and environmental friendliness. The composition of $\mathrm{MnO}_{2}$ materially changes the morphology of the surface and leads to an increase in the pseudocapacitive performance of $\mathrm{MnO}_{2}$ as an increase in porosity [2]. The proposed theoret- ical reasons for $\mathrm{MnO}_{2}$ being a better alternative for electrode materials is its high theoretical capacity in reference to an individual electron-based redox potential reactions of each atom was observed in a wider potential window, electroactive in neutral electrolytes, etc. [10]. These lead to lower corrosion of the collector, and other benefits were incorporated in Figure 1. Improving the performance of electrode materials for the production of high-energy supercapacitors.

The availability of the material is also found to be in excess naturally, which decreases its price value. The crystal structure of the metal also forms crystallinity hence supporting charge storage and dissipation. It has been reported that tunnel or chain structure of alpha, beta, and gamma $\mathrm{MnO}_{2}$ facilitates easier transfer of electrons with higher capacitance. Nanoranged $\mathrm{MnO}_{2}$ has a larger reaction area for feasible and enhanced cation intercalation and deintercalation when compared to its amorphous form [11, 12].

In recent years, graphene attained huge interest especially in the fields of electric devices, energy storage applications, and sensors due to their unique property of physiochemical characteristics, such as high surface area, excellent conductivity, and mechanical stability. Structurally, graphene oxide (GO) has been visualized as a graphene sheet with its basal plane and edges filled with oxygen groups. Graphene hybridization with functional NMs enhances the component functional properties and even produces new properties through cooperative interaction.

$\mathrm{GO}$ has been an appropriate support material for $\mathrm{MnO}_{2}$ loadings in electroactive materials for supercapacitors [13]. GO not only affords high surface area for $\mathrm{MnO}_{2}$ nanosheets deposition but also provides to those nanostructures good adhesion. GO had a significant impression on the property of electrochemical in the $\mathrm{GO}-\mathrm{MnO}_{2}$ nanocomposites. Scheme of $\mathrm{MnO}_{2}$ nanocomposites shown in Figure 2 mentioned with the application of supercapacitors made out of $\mathrm{MnO}_{2}$ with optimizing parameters which helps in improving the capacitance, stability, and storage etc.

$\mathrm{MnO}_{2}$ nanoparticles can be deposited uniformly on GO with high density. The high loading efficiency of $\mathrm{MnO}_{2}$ and high surface area of $\mathrm{MnO}_{2}$-GO increase the specific capacitance of $\mathrm{MnO}_{2}-\mathrm{GO}$ [14].

This work is aimed at fabricating the composite of $\mathrm{MnO}_{2}$-GO and achieving good conductivity in the charge storage process. The $\mathrm{MnO}_{2}$ and $\mathrm{MnO}_{2}-\mathrm{GO}$ composites were synthesized by the electrodeposition method. After electrodeposition, the galvanostatic charge-discharge analysis was performed to characterize the charge storage ability of the film from which we can find the ability of the composite as an electrode of a supercapacitor.

\section{Materials and Method}

2.1. Fabrication of $\mathrm{MnO}_{2}-\mathrm{GO}$ Nanocomposite Supercapacitor. $\mathrm{MnO}_{2}$ was synthesized using standard protocol reported previously in 2015 [15]. Graphene oxide was synthesized by modified Hummers method [16]. $\mathrm{MnO}_{2}$ and $\mathrm{MnO}_{2}-\mathrm{GO}$ NMs were coated on electricity collector substrates by constant potentiostat mode in the electrodeposition method. The SS substrate was cut and polished into 


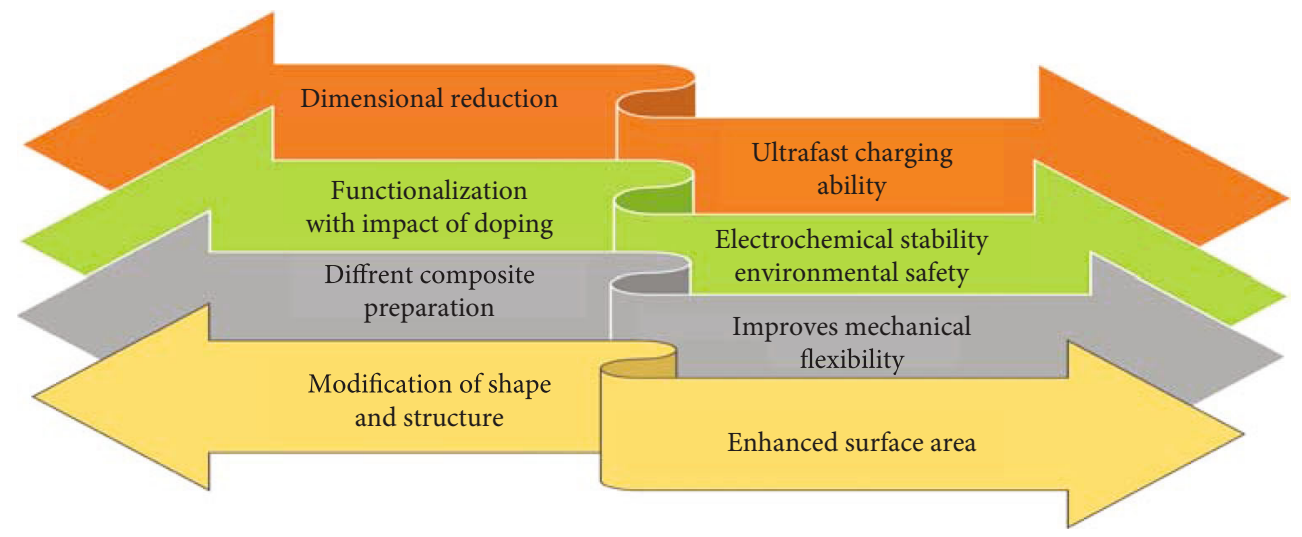

FIGURE 1: Performance enhancement of electrode materials for high-energy supercapacitors.
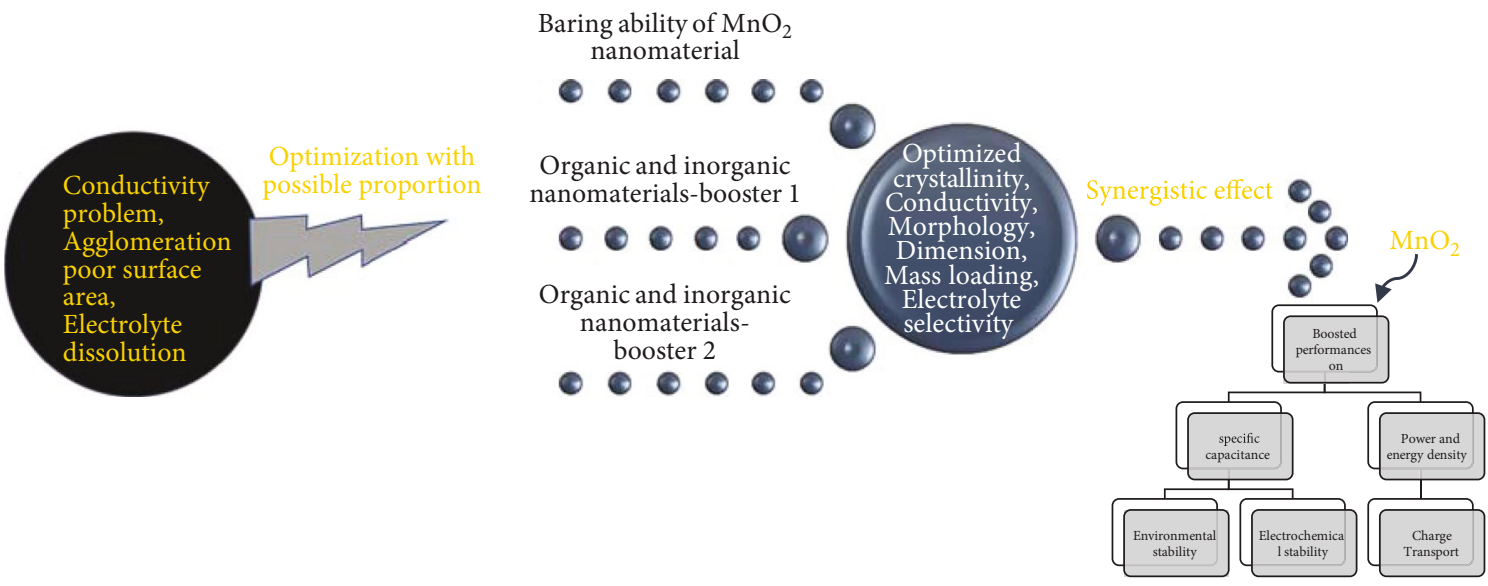

FIgURE 2: Scheme of $\mathrm{MnO}_{2}$ nanocomposites for the application of supercapacitors.

$1 \times 1 \mathrm{~cm}$ size and then washed for $15 \mathrm{~min}$ in deionized water by a bath sonicator.

The electrochemical deposition was performed for $30 \mathrm{~min}$ at $0.8 \mathrm{~V}$ using the as prepared SS substrate as the working electrode. Different concentrations of GO $(10 \mathrm{mg}$, $15 \mathrm{mg}$, and $20 \mathrm{mg}$ ) with $25 \mathrm{ml}$ DI water were dispersed by ultrasonication, and then $0.1 \mathrm{M}$ of $\mathrm{MnSO}_{4}$ solution was added to prepare the electrolyte solution. The $\mathrm{pH}$ of the electrolyte solution was maintained at 10 by adding $1 \mathrm{M} \mathrm{NaOH}$. $\mathrm{Ag} / \mathrm{AgCl}$ and Pt electrodes were used as reference and counter electrodes, respectively [17]. The resulting thin films were dried at room temperature after the process of rinsing in DI water. The overall process of synthesis was displayed in the scheme of Figure 3.

\subsection{Electrochemical Analysis}

2.2.1. Analyzing Setup. For the electrochemical analyses $0.1 \mathrm{M}$ of $\mathrm{Na}_{2} \mathrm{SO}_{4}$ dissolved in $50 \mathrm{ml}$ of double-distilled water used as an electrolyte solution, $\mathrm{Ag} / \mathrm{AgCl}$ was fixed as the reference electrode, whereas prepared $\mathrm{MnO}_{2}$ electrodes made with thin films are considered as working electrodes, and electrodes made out of platinum wire are considered as a counter electrode. These three electrodes were immersed in the electrolyte solution the setup was connected to the BIO-
LOGIC Science SP-50 model Electrochemical Workstation for the analyses.

2.2.2. Cyclic Voltammetry. The electrochemical characteristics of specified composites are typically measured using cyclic voltammetry, a standard technique. The potential scan starts at a point where there is no electrochemical reaction. The scan proceeds to the switching potential at a predetermined constant rate, then reverses direction and returns to the electrode as oxidation or reduction, accordingly. The formula below was used to compute the active material's specific capacitance.

$$
\text { Specific capacitance }=C=\frac{I}{\left(m v\left(V_{a}-V_{c}\right)\right.}\left(\frac{F}{g}\right) \text {. }
$$

$I$ considered as applied current $(\mathrm{A})$, where $\left(V_{a}-V_{c}\right)$ are considered as the potential of sweep window $(\mathrm{V}), m$ is known as mass of the active material $(\mathrm{g})$, and $v$ is the scan rate $\left(\mathrm{Vs}^{-1}\right)$.

2.2.3. Galvanostatic Charge-Discharge. The charge-discharge potential and cycle ability of prepared $\mathrm{MnO}_{2}-\mathrm{GO}$ nanocomposite was measured using galvanostatic charge-discharge 


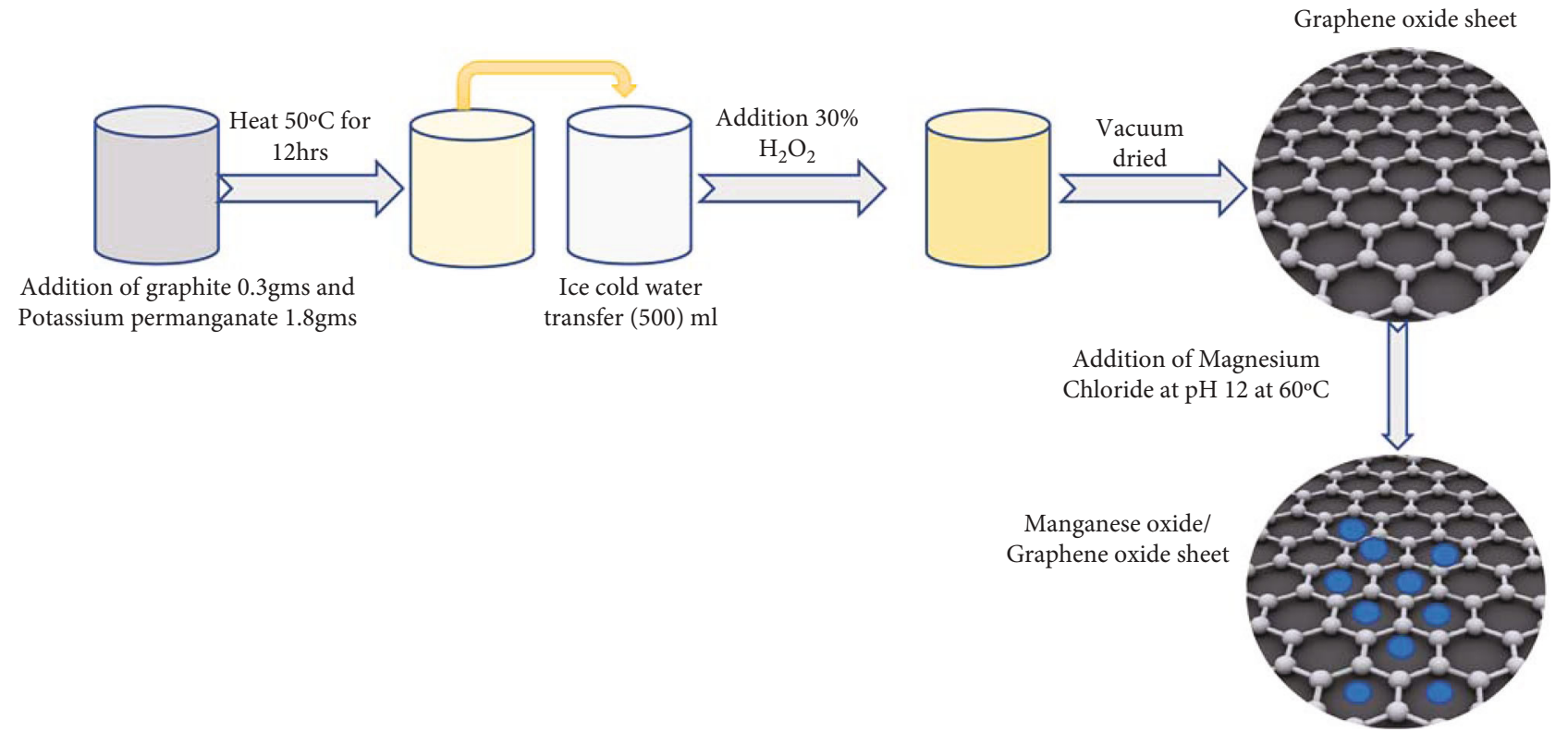

FIgure 3: Schematic of GO sheets and formation of $\mathrm{MnO}$ /GO nanocomposites.

(GCD) technique. It is necessary to conduct the charge and discharge at a constant current until a predetermined voltage is obtained. A cycle is a loop of charging and discharging that repeats over and over again. The time required to reach the set potentials for the applied current was plotted. In the charging process, the constant rate of increment in the potential and the discharge process the constant rate of decrement in the potential with time is the perfect behavior for the charge storage device.

Using the charge-discharge curve the capacitance and cycling behavior of the electrode material has been monitored, and also the charge density, energy density, and capacitance of the material were calculated from the following equations.

Specific Capacitance of the active material is $C=\frac{(I X \Delta t / \Delta V X m) F}{g}$.

\section{Results and Discussions}

3.1. Cyclic Voltammetry Studies. Cyclic voltammetry was performed using prepared $\mathrm{MnO}_{2}$ and $\mathrm{MnO}_{2}-\mathrm{GO}$ thin films. Here, prepared thin films were fixed as working electrodes whereas the platinum wire was considered as a counter electrode, and $\mathrm{Ag} / \mathrm{AgCl}$ was a reference electrode, respectively, an aqueous solution of $0.1 \mathrm{M} \mathrm{Na}_{2} \mathrm{SO}_{4}$ as the electrolyte for different scan rates.

Figure 4 shows a cyclic voltammogram of $\mathrm{MnO}_{2}$ and $\mathrm{MnO}_{2}$-GO thin films by applying a scan rate of $5 \mathrm{mV} \mathrm{s}^{-1}$ between the potential of 0 and $0.8 \mathrm{~V}$. It shows the current density vs. potential curve of $\mathrm{MnO}_{2}$ : $\mathrm{GO}$ (different concentration) composites and pure $\mathrm{MnO}_{2}$. From that, we found that $\mathrm{MnO}_{2}$ has a low current density $10 \mathrm{mg} \mathrm{MnO}_{2}$ added for GO composite which has high current density compared

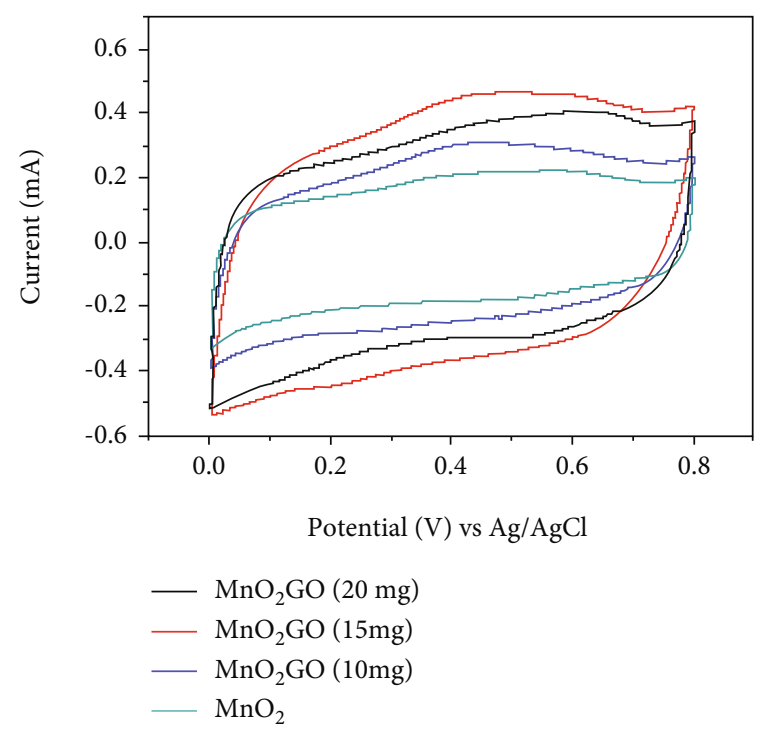

FIgure 4: CV current density of $\mathrm{MnO}_{2}$ and $\mathrm{MnO}_{2}$-GO.

to pure $\mathrm{MnO}_{2}$. Also, $15 \mathrm{mg} \mathrm{MnO}_{2}$-GO composite has a high current density compared to $10 \mathrm{mg}$ composite, and $20 \mathrm{mg}$ $\mathrm{MnO}_{2}-\mathrm{GO}$ composite has a low current density compared to $15 \mathrm{mg}$ composite because further increment in GO tends to decrease the amount of $\mathrm{MnO}_{2}$ on the surface of the electrode. Ding reported successful electrodeposition of manganese dioxide nanoparticles onto an indium tin oxide glass substrate using the cyclic voltammetry (CV) method, which was used to create an indium tin oxide glass substrate from an aqueous solution of $0.1 \mathrm{M} \mathrm{Na}_{2} \mathrm{SO}_{4}$ containing $5 \times 10^{-3}$ $\mathrm{M} \mathrm{MnSO}_{4}$, and they achieved $294 \mathrm{Fg}^{-1}$ [9].

3.2. Galvanostatic Charge-Discharge Technique. Galvanostatic charge/discharge experiments were performed to evaluate 


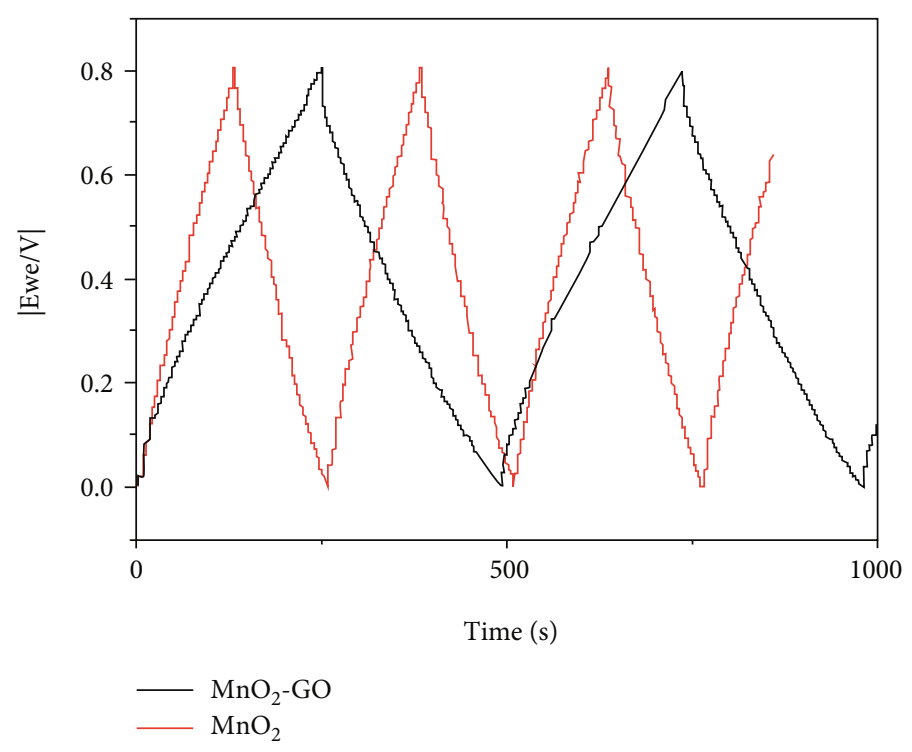

Figure 5: Charge and discharge times in $\mathrm{MnO}_{2}$ and $\mathrm{MnO}_{2}$-GO.

the specific capacitance and cycle ability of the developed $\mathrm{MnO}_{2}$ and $\mathrm{MnO}_{2}-\mathrm{GO}$ electrode materials. Further, the insight into the relationship between the specific capacitance of the different $\mathrm{MnO}_{2}$ nanostructures has been studied. The galvanostatic charge-discharge is also performed using the biologic sp-50 electrochemical workstation. Figure 5 depicts the charge-discharge performance of the electrodeposited $\mathrm{MnO}_{2}$ for the first few cycles.

The potential-time curve was measured from 0 to $0.8 \mathrm{~V}$ versus the reference electrode for more than 300 cycles, $0.1 \mathrm{M} \mathrm{Na}_{2} \mathrm{SO}_{4}$ was used at a current density of $0.5 \mathrm{~mA} \mathrm{~cm}^{-}$ ${ }^{2}$. The curves are almost linear and present in the form of typical symmetrical triangle shape which indicates the pseudocapacitance of $\mathrm{MnO}_{2}$. The composition of $\mathrm{MnO}_{2}$-GO has a high charge and discharge time compared to $\mathrm{MnO}_{2}$. Thus, it reported to have increased specific capacitance of $\mathrm{MnO}_{2}$ GO. Sebastin et al. have reported that multilayered film electrodes show good electrochemical properties [6]. Hence, the obtained results indicate that these newly synthesized films could be used as potential applications in electrochemical capacitors.

Figure 6 clearly shows the variation in specific capacitance with respect to the cycle number of $\mathrm{MnO}_{2}$ and $\mathrm{MnO}_{2}$-GO electrodes. In which $\mathrm{MnO}_{2}$ has the specific capacitance of $80 \mathrm{~F} / \mathrm{g}$ for the first cycle whereas $\mathrm{MnO}_{2}-\mathrm{GO}$ has the specific rate of capacitance up to $140 \mathrm{~F} / \mathrm{g}$ for first cycle. $\mathrm{MnO}_{2}$-GO displays high specific capacitance compared with $\mathrm{MnO}_{2}$. Furthermore, the linear line indicates the cycling performance of $\mathrm{MnO}_{2}$ and $\mathrm{MnO}_{2}$-GO. It shows the films have retained almost $95 \%$ of their specific capacitance even after 300 cycles. With these promising results, these materials can be used as an ideal material in supercapacitor applications.

Addition of $\mathrm{MnO}_{2}$ to graphene oxide sheets addresses the low volumetric density of graphene-based electrodes. This would prevent the agglomeration of the sheets which happens due to Van der Waals attraction between the nano-

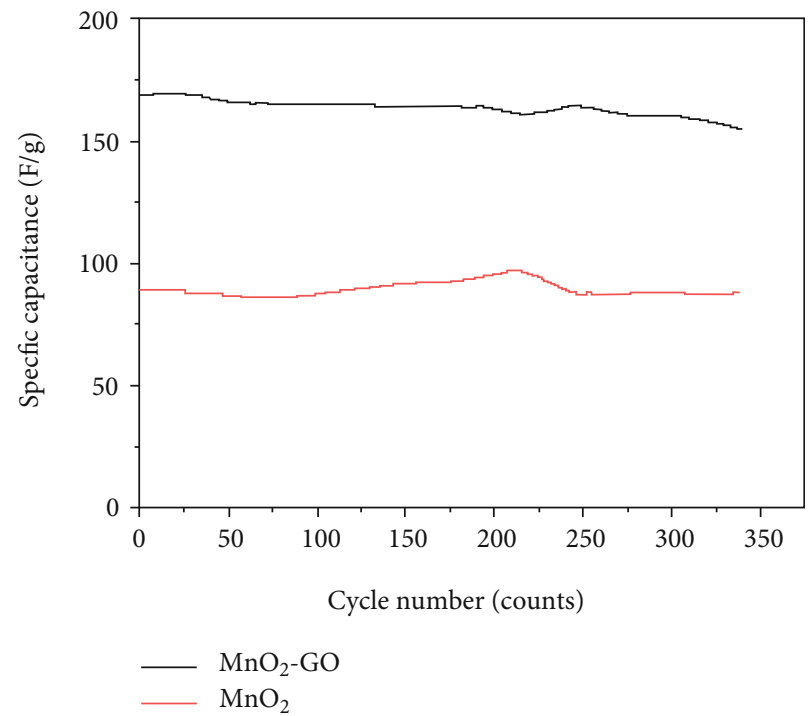

Figure 6: Cycling ability of $\mathrm{MnO}_{2}$ and $\mathrm{MnO}_{2}-\mathrm{GO}$.

particle and the porous material [18]. Elastic constraint is provided by the carbon skeleton of GO. This avoids the dissolution of the electroactive property of $\mathrm{MnO}_{2}$. A simple microwave-assisted technique can rapidly green synthesize $\mathrm{MnO}_{2} / \mathrm{GO}$ composites by simple deposition of the nanoparticle onto the sheet [19]. Previous studies stated that doping of $\mathrm{MnO}_{2}$ increases the specific capacitance by three times when compared to pure GO sheet or pure birnessite $\mathrm{MnO}_{2}$ $[19,20]$.

The ratio of the $\mathrm{MnO} 2$ by weight plays a crucial role in the capacitance performance. With an increasing mass ratio, the specific capacitance tends to increase which coincides with the results we have obtained for the current study [21]. Composites tend to have higher diffusivity and mobility of charges than disordered $\mathrm{MnO} 2$. Changing the crystal structures and orientations of $\mathrm{MnO} 2$, it is suggested that 
TABLE 1: Reported outcomes on $\mathrm{MnO} / \mathrm{GO}$ composites for supercapacitor applications.

\begin{tabular}{|c|c|c|c|}
\hline Electrolyte & Crystal/porous material & Specific capacitance & Reference \\
\hline $1 \mathrm{M} \mathrm{Na}_{2} \mathrm{SO}_{4}$ & Gamma $\mathrm{MnO}_{2}$ crystal with graphene & $270\left(0.5 \mathrm{~A} \mathrm{~g}^{-1}\right)$ & {$[24]$} \\
\hline $1 \mathrm{M} \mathrm{KOH}$ & $\mathrm{MnO}_{2}$ or graphene & $342.8\left(0.5 \mathrm{~A} \mathrm{~g}^{-1}\right)$ & {$[25]$} \\
\hline $1 \mathrm{M} \mathrm{Na}_{2} \mathrm{SO}_{4}$ & $\mathrm{~N}$ doped graphene $/ \mathrm{MnO}_{2}$ & $411.5\left(0.5 \mathrm{~A} \mathrm{~g}^{-1}\right)$ & {$[26]$} \\
\hline $\mathrm{Na}_{2} \mathrm{SO}_{4} 1 \mathrm{M}$ & Graphene oxide/ $\mathrm{MnO}_{2}$ & $315\left(0.5 \mathrm{~A} \mathrm{~g}^{-1}\right)$ & {$[27]$} \\
\hline $\mathrm{Na}_{2} \mathrm{SO}_{4} 1 \mathrm{M}$ & Graphene oxide $/ \mathrm{MnO}_{2}$ & $360.3\left(0.5 \mathrm{~A} \mathrm{~g}^{-1}\right)$ & {$[28]$} \\
\hline $\mathrm{Na}_{2} \mathrm{SO}_{4} 1 \mathrm{M}$ & Reduced graphene oxide $/ \mathrm{MnO}_{2}$ & $759\left(2 \mathrm{~A} \mathrm{~g}^{-1}\right)$ & [29] \\
\hline $1 \mathrm{M} \mathrm{Na}_{2} \mathrm{SO}_{4}$ & Graphene $/ \mathrm{MnO}_{2}$ & $234.8\left(0.1 \mathrm{~A} \mathrm{~g}^{-1}\right)$ & {$[30]$} \\
\hline $1 \mathrm{M} \mathrm{Na}_{2} \mathrm{SO}_{4}$ & Graphene/ $\mathrm{MnO}_{2}$ & $255\left(0.5 \mathrm{~A} \mathrm{~g}^{-1}\right)$ & {$[31]$} \\
\hline $1 \mathrm{MNa}_{2} \mathrm{SO}_{4}$ & Graphene $/ \mathrm{MnO}_{2}$ & $133\left(0.5 \mathrm{mvs}^{-1}\right)$ & {$[32]$} \\
\hline $1 \mathrm{M} \mathrm{Na}_{2} \mathrm{SO}_{4}$ & Graphene/CNT/ $\mathrm{MnO}_{2}$ & $372\left(0.5 \mathrm{~A} \mathrm{~g}^{-1}\right)$ & [33] \\
\hline $1 \mathrm{M} \mathrm{Na}_{2} \mathrm{SO}_{4}$ & Sponge reduced graphene oxide/ $\mathrm{MnO}_{2}$ & $205\left(0.1 \mathrm{~A} \mathrm{~g}^{-1}\right)$ & {$[20]$} \\
\hline- & Graphene/ $\mathrm{MnO}_{2}$ & $310\left(2 \mathrm{mvs}^{-1}\right)$ & {$[34]$} \\
\hline- & $\mathrm{MnO}_{2} / \mathrm{CNP} /$ graphene & $255\left(2 \mathrm{mvs}^{-1}\right)$ & [35] \\
\hline- & Graphene/MnO2/activated carbon fiber felt & $* 1.516$ & {$[36]$} \\
\hline- & Reduced graphene oxide- $\mathrm{MnO}_{2}$ & $274\left(10 \mathrm{mvs}^{-1}\right)$ & [37] \\
\hline- & $\mathrm{Mn}_{3} \mathrm{O}_{4} / \mathrm{rGO}$ & $* * 52.2$ & {$[38]$} \\
\hline $1 \mathrm{M} \mathrm{Na}_{2} \mathrm{SO}_{4}$ & $\mathrm{GO} / \mathrm{MnO}_{2}$ & $140\left(0.5 \mathrm{~A} \mathrm{~g}^{-1}\right)$ & Current study \\
\hline
\end{tabular}

$\mathrm{GO} / \mathrm{MnO} 2$ performance as an active material can be greatly influenced. Eco-friendly techniques under milder conditions are found to be more feasible to control the shape and dimension of the nanoparticle [22]. Controlling oxygen functional moieties and doping of heteroatom on the GO sheets can aid in manufacturing hi-performance electrode materials.

Synthesis method has a great influence on the electrochemical performance of $\mathrm{MnO}_{2} / \mathrm{GO}$ nanocomposites. The specific capacitance of current study is quite low when compared to previously reported nanocomposites. We infer that the microwave technique opted by might not have supported the formation of proper crystal formation in $\mathrm{MnO}_{2}$. As it was already reported that crystal structure positively influences the specific capacitance of a capacitor material. The nanoparticle used in this study does provide high energy density to the porous GO, but the structure of the lattice might not be at its very best. GO ensures upgraded cyclic performance and stability. The microstructure of the sheets with functional groups portrays different properties in various orientations and dimensions for the given nanocomposite. This gives the rational feasibility to optimize this nanocomposite and maximize it to its potential. Nanotubes and nanofibers offer high and rapid diffusion due to its one dimensional orientation.

This shortens the ion diffusion pathway which leads to high conductivity and enhanced mechanical properties. Flexibility is also attained by the capacitor material. Similarly, two-dimensional GO is also regarded as an ideal conductive substrate with enhanced specific surface area, conductivity, and ultralow density. Compared to nanotubes, the diffusivity is quite low for GO. Activated form of carbon is mostly opted for fabrication of storage devices owing to its three-dimensional structure and rich functional groups. Although a rich porous support structure is provided in the current study for the nanoparticle the performance yielded was quite less. Orderly channels without obstructions can further maximize the electrochemical performance of $\mathrm{MnO}$. They accelerate the ion transport and make the diffusivity easier for $\mathrm{MnO}_{2}$.

Hence, the current material can be experimented with by incorporating additional channels in the Go sheet to see if the performance could be improved. Even the graphitization of the sheet makes conducive improvement in the transfer of charge between the nanoparticle and the carbon base. Low graphitization of the sheet used in the current study might also be the reason for low performance of the capacitor material. In the previous years of effort has been taken to explore the potential of $\mathrm{MnO}_{2} / \mathrm{GO}$ composites for their capacitance function. Exciting outcomes have been accomplished so far. There are still many drawbacks in the nanoparticle that hinder its electrochemical property if not set right. A few have already been discussed above [23]. Efforts need to be taken to focus on developing new generation nanoparticle combinations with potential doping to address all drawbacks mentioned above. Table 1 represents the previously reported outcomes on $\mathrm{MnO}_{2} / \mathrm{GO}$ composites for supercapacitor applications.

\section{Limitations and Future Scope}

A greater amount of testing and research into $\mathrm{MnO} 2-\mathrm{GO}$ materials is required in order to produce better supercapacitors in the future. Aspects such as poor structural stability and reduced ion diffusion need to be addressed as well [38-44]. Researchers should look into and test highcapacitance materials that can be reversed and modified, when necessary, as well as rapid cation diffusion at high charging and discharging rates. 


\section{Conclusion}

An electrochemical deposition approach was used to deposit $\mathrm{MnO}_{2}$ and varied concentrations of $\mathrm{MnO}_{2}$-GO composites on an SS substrate in this investigation. The CV curves depicted the electrochemical performance of pure and composite materials synthesized in various ratios. In comparison to pure, $10 \mathrm{mg}$, and $20 \mathrm{mg} \mathrm{GO}$ added $\mathrm{MnO} 2$ thin films, the $15 \mathrm{mg}$ GO- $\mathrm{MnO}_{2}$ electrodeposited film has a high current density. Because it covers the highest area of $\mathrm{MnO} 2$ in the composite, increasing the GO tends to lower the film's performance. The charge storage behavior of the films is demonstrated by galvanostatic charge-discharge analysis, galvanostatic charge-discharge curves have been conducted with different composition of GO to ensure the effective capacitance which shows that $\mathrm{MnO} 2$ and $\mathrm{MnO}_{2}-\mathrm{GO}$ thin films have specific capacitances of $80 \mathrm{~F} / \mathrm{g}$ and $140 \mathrm{~F} / \mathrm{g}$ for the first cycle, respectively, and that the composite retains approximately 95 percent of capacitance after 300 cycles. When compared to previously published nanocomposites, the current study's specific capacitance is quite low. We deduce that the microwave approach used may not have promoted the creation of appropriate $\mathrm{MnO}_{2}$ crystals. Despite the fact that the nanoparticle was given a rich porous support structure in the current work, the results were disappointing. $\mathrm{MnO}_{2}$ electrochemical performance can be improved even further by having clean, unobstructed channels. Although the nanoparticle utilized in this study provides a high energy density to the porous GO, the lattice structure may not be optimal. Low graphitization of the sheet utilized in this investigation could possibly be a factor in the capacitor's poor performance.

\section{Data Availability}

The data used to support the findings of this study are included in the article.

\section{Conflicts of Interest}

The authors declare that there is no conflict of interest regarding the publication of this article.

\section{Acknowledgments}

Alagar Karthick gratefully acknowledges group FQM-383 from Universidad de Cordoba, Spain for the provision of a honorary visiting research position in the group. This project was funded by the Researchers Supporting Project number (RSP-2021/405), King Saud University, Riyadh, Saudi Arabia.

\section{References}

[1] S. Chen, L. Qiu, and H. M. Cheng, "Carbon-based fibers for advanced electrochemical energy storage devices," Chemical Reviews, vol. 120, no. 5, pp. 2811-2878, 2020.

[2] D. Rajesh, M. K. Francis, P. B. Bhargav, A. Nafis, and C. Balaji, "2D layered nickel-cobalt double hydroxide nano sheets @ 1D silver nanowire- graphitic carbon nitrides for high perfor- mance super capacitors," Journal of Alloys and Compounds, p. 162803, 2021.

[3] N. Hemalatha, R. Saravanakumar, P. Veeramanikandan, and V. R. Kumar, "Evaluation of capacitance cycle ability with $\mathrm{MnO} 2$-go nanocomposite supercapacitor," in 2021 6th International Conference on Communication and Electronics Systems (ICCES), pp. 102-106, 2021.

[4] A. K. Priya, R. Gokulan, A. Vijayakumar, and S. Praveen, "Biodecolorization of remazol dyes using biochar derived from ulva reticulata: isotherm, kinetics, desorption, and thermodynamic studies," Desalination and Water Treatment, vol. 200, pp. 286-295, 2020.

[5] V. Yogeshwaran and A. K. Priya, "Experimental studies on the removal of heavy metal ion concentration using sugarcane bagasse in batch adsorption process," Desalination and Water Treatment, vol. 224, p. 27160, 2021.

[6] S. Sebastin, A. K. Priya, A. Karthick, R. Sathyamurthy, and A. Ghosh, "Agro waste sugarcane bagasse as a cementitious material for reactive powder concrete," Clean Technology, vol. 2, pp. 476-491, 2020.

[7] D. Salinas-Torres, R. Ruiz-Rosas, E. Morallón, and D. CazorlaAmorós, "Strategies to enhance the performance of electrochemical capacitors based on carbon materials," Front. Mater., vol. 6, p. 115, 2019.

[8] B. E. Conway, Electrochemical Supercapacitors: Scientific Fundamentals and Technological Applications, Springer Science \& Business Media, 2013.

[9] K. Q. Ding, "Cyclic voltammetrically prepared copperdecorated $\mathrm{MnO} 2$ and its electrocatalysis for oxygen reduction reaction (ORR)," International Journal of Electrochemical Science, vol. 5, pp. 72-78, 2009.

[10] Y. Xie, C. Yang, P. Chen, D. Yuan, and K. Guo, “ $\mathrm{MnO}_{2}$-decorated hierarchical porous carbon composites for highperformance asymmetric supercapacitors," Journal of Power Sources, vol. 425, pp. 1-9, 2019.

[11] M. Yang and K. L. Choy, "A nature-derived, flexible and three dimensional (3D) nano-composite for chronic wounds $\mathrm{pH}$ monitoring," Materials Letters, vol. 288, article 129335, 2021.

[12] J. R. Rajabathar, H. A. Al-lohedan, P. Arunachalam et al., "Synthesis and characterization of metal chalcogenide modified graphene oxide sandwiched manganese oxide nanofibers on nickel foam electrodes for high performance supercapacitor applications," Journal of Alloys and Compounds, vol. 850, article 156346, 2021.

[13] Y. Liu, D. Yan, Y. Li et al., "Manganese dioxide nanosheet arrays grown on graphene oxide as an advanced electrode material for supercapacitors," Electrochimica Acta, vol. 117, pp. 528-533, 2014.

[14] C. Ruttanapun, C. Phrompet, W. Tuichai, A. Karaphun, S. Daengsakul, and C. Sriwong, "Influence of free electron charge and free extra framework anions in calcium email protected rGO (email protected rGO) cement composites with enhanced dielectric and electrochemical properties," Journal of the Taiwan Institute of Chemical Engineers, vol. 127, pp. 334-348, 2021.

[15] H. Aminirastabi, Z. Weng, H. Xue et al., "Evaluation of nano grain growth of $\mathrm{TiO}_{2}$ fibers fabricated via centrifugal jet spinning," Nano-Structures \& Nano-Objects, vol. 21, article 100413, 2020.

[16] T. Nibedita Dey, "A Comparative evaluation of graphene oxide based materials for electrochemical non-enzymatic sensing of 
curcumin," Materials Research Express, vol. 5, no. 2, article 025406, 2018.

[17] Suhasini, "Effect of deposition method and the surfactant on high capacitance of electrochemically deposited $\mathrm{MnO}_{2}$ on stainless steel substrate," Journal of Electroanalytical Chemistry, vol. 690, pp. 13-18, 2013.

[18] L. Sheng, L. Jiang, T. Wei, and Z. Fan, "High volumetric energy density asymmetric supercapacitors based on well-balanced graphene and Graphene-MnO2Electrodes with densely stacked architectures," Small, vol. 12, no. 37, pp. 5217-5227, 2016.

[19] Z. Yang, J. Zhang, M. C. W. Kintner-Meyer et al., "Electrochemical energy storage for green grid," Chemical Reviews, vol. 111, pp. 3577-3613, 2011.

[20] J. Yan, Z. Fan, T. Wei, W. Qian, M. Zhang, and F. Wei, "Fast and reversible surface redox reaction of graphene- $\mathrm{MnO} 2 \mathrm{com}$ posites as supercapacitor electrodes," Carbon, vol. 48, pp. 3825-3833, 2010.

[21] S. Saha, P. Maji, D. A. Pethsangave et al., "Effect of morphological ordering on the electrochemical performance of $\mathrm{MnO} 2$ graphene oxide composite," Electrochimica Acta, vol. 317, pp. 199-210, 2019.

[22] G. Zhu, Z. He, J. Chen et al., "Highly conductive threedimensional $\mathrm{MnO} 2$-carbon nanotube-graphene-Ni hybrid foam as a binder-free supercapacitor electrode," Nanoscale, vol. 6, pp. 1079-1085, 2014.

[23] T. Wang, D. Song, H. Zhao et al., "Facilitated transport channels in carbon nanotube/carbon nanofiber hierarchical composites decorated with manganese dioxide for flexible supercapacitors," Journal of Power Sources, vol. 274, pp. 709717, 2015.

[24] X. Wang, L. Chen, S. Zhang et al., "Compounding $\delta$-MnO2 with modified graphene nanosheets for highly stable asymmetric supercapacitors," Colloids and Surfaces, vol. 573, pp. 57-66, 2019.

[25] H. Wang, Q. Fu, and C. Pan, "Green mass synthesis of graphene oxide and its $\mathrm{MnO} 2$ composite for high performance supercapacitor," Electrochimica Acta, vol. 312, pp. 11-21, 2019.

[26] Q. Le, M. Huang, T. Wang, X. Liu, L. Sun, and X. Guo, "Biotemplate derived three dimensional nitrogen doped graphene@MnO2 as bifunctional material for supercapacitor and oxygen reduction reaction catalyst," Journal of Colloid and Interface Science, vol. 544, pp. 155-163, 2019.

[27] Z. S. Wu, W. Ren, D. W. Wang, F. Li, B. Liu, and H. M. Chen, "High-energy $\mathrm{MnO} 2$ nanowire/graphene and graphene asymmetric electrochemical capacitors," ACS Nano, vol. 4, pp. 5835-5842, 2010.

[28] K. Dai, L. Lu, C. Liang, J. Dai, Q. Liu, and Y. Zhang, "In situ assembly of $\mathrm{MnO} 2$ nanowires/graphene oxide nanosheets composite with high specific capacitance," Electrochimica Acta, vol. 116, pp. 111-117, 2014.

[29] S. Jadhav, R. S. Kalubarme, C. Terashima, B. B. Kale, V. Godbole, and A. Fujishima, "Manganese dioxide/reduced graphene oxide composite an electrode material for highperformance solid state supercapacitor," Electrochimica Acta, vol. 299, pp. 34-44, 2019.

[30] Y. Cheng, S. Lu, H. Zhang, C. V. Varanasi, and J. Liu, "Synergistic effects from graphene and carbon nanotubes enable flexible and robust electrodes for high-performance supercapacitors," Nano Letters, vol. 12, pp. 4206-4211, 2012.
[31] W. S. Li, M. L. Chang, K. C. Chuang, Y. S. Li, J. D. Luo, and H. C. Cheng, "Electrochemical properties of $\mathrm{CNT} / \mathrm{MnO} 2$ hybrid nanostructure with low-temperature hydrothermal synthesis as high-performance supercapacitor," Journal of the Electrochemical Society, vol. 166, pp. A2194-A2198, 2019.

[32] R. Amade, A. Muyshegyan-Avetisyan, J. M. González, A. P. Pino, E. György, and E. Pascual, "Super-capacitive performance of manganese dioxide/graphene nano-walls electrodes deposited on stainless steel current collectors," Materials, vol. 12, p. 483, 2019.

[33] J. Ge, H. B. Yao, W. Hu, X. F. Yu, Y. X. Yan, and L. B. Mao, "Facile dip coating processed graphene/ $\mathrm{MnO} 2$ nanostructured sponges as high performance supercapacitor electrodes," Nano Energy, vol. 2, pp. 505-513, 2013.

[34] Y. Xiong, M. Zhou, H. Chen et al., "Synthesis of honeycomb $\mathrm{MnO}_{2}$ nanospheres/carbon nanoparticles/graphene composites as electrode materials for supercapacitors," Applied Surface Science, vol. 357, pp. 1024-1030, 2015.

[35] Q. Yang, L. Dong, C. Xu, and F. Kang, "High-performance supercapacitors based on graphene/MnO2/activated carbon fiber felt composite electrodes in different neutral electrolytes," RSC Advances, vol. 6, no. 15, pp. 12525-12529, 2016.

[36] G. Zhang, L. Ren, L. Deng, J. Wang, L. Kang, and Z.-H. Liu, "Graphene- $\mathrm{MnO}_{2}$ nanocomposite for high-performance asymmetrical electrochemical capacitor," Materials Research Bulletin, vol. 49, pp. 577-583, 2014.

[37] T. Xiong, W. S. V. Lee, X. Huang, and J. M. Xue, "Mn3O4/ reduced graphene oxide based supercapacitor with ultra-long cycling performance," Journal of Materials Chemistry A, vol. 5, no. 25, pp. 12762-12768, 2017.

[38] J. G. Wang, Y. Yang, Z. H. Huang, and F. Kang, "Effect of temperature on the pseudo-capacitive behavior of freestanding MnO2@carbon nanofibers composites electrodes in mild electrolyte," Journal of Power Sources, vol. 224, pp. 86-92, 2013.

[39] T. Yousefi, A. N. Golikand, M. H. Mashhadizadeh, and M. Aghazadeh, "Template-free synthesis of $\mathrm{MnO} 2$ nanowires with secondary flower like structure: characterization and supercapacitor behavior studies," Current Applied Physics, vol. 12, no. 1, pp. 193-198, 2012.

[40] Y. Qian, S. Lu, and F. Gao, "Preparation of $\mathrm{MnO} 2$ /graphene composite as electrode material for supercapacitors," Journal of Materials Science, vol. 46, no. 10, pp. 3517-3522, 2011.

[41] Z. Li, Y. Mi, X. Liu, S. Liu, S. Yang, and J. Wang, "Flexible graphene/MnO2 composite papers for supercapacitor electrodes," Journal of Materials Chemistry, vol. 21, no. 38, pp. 1470614711, 2011.

[42] J. Zhang, J. Jiang, and X. S. Zhao, "Synthesis and capacitive properties of manganese oxide nanosheets dispersed on functionalized graphene sheets," The Journal of Physical Chemistry C, vol. 115, no. 14, pp. 6448-6454, 2011.

[43] L. Zhang, R. Jamal, Q. Zhao, M. Wang, and T. Abdiryim, "Preparation of PEDOT/GO, PEDOT/MnO2, and PEDOT/ $\mathrm{GO} / \mathrm{MnO} 2$ nanocomposites and their application in catalytic degradation of methylene blue," Nanoscale Research Letters, vol. 10, no. 1, pp. 1-9, 2015.

[44] J. Wang, R. Zeng, D. Guodong et al., "Rapid microwaveassisted synthesis of various $\mathrm{MnO}_{2}$ nanostructures and their magnetic properties," Materials Chemistry and Physics, vol. 166 , no. 15 , pp. $42-48,2015$. 